www.jmscr.igmpublication.org

Impact Factor 5.84

Index Copernicus Value: 71.58

ISSN (e)-2347-176x ISSN (p) 2455-0450

crossref DOI:

Journal Of Medical Science And Clinical Research

\title{
A Study on Prevalence, Risk Factors of Multi Drug Resistant Organism and its Impact in Diabetic Foot
}

\section{Dr B.Deni Raja ${ }^{1 *}$, Dr V.R.Baskaran ${ }^{2}$, Dr V.Natarajan ${ }^{3}$, Dr R.Renjith Singh ${ }^{4}$}

${ }^{* 1,4}$ Post Graduate, Department of General Surgery, Rajah Muthiah Medical College and Hospital, Annamalai University, Annamalai Nagar - 608002

${ }^{2}$ Professor, Department of General Surgery, Rajah Muthiah Medical College and Hospital, Annamalai University, Annamalai Nagar - 608002

${ }^{3}$ Professor, Department of Microbiology, Rajah Muthiah Medical College and Hospital,

Annamalai University, Annamalai Nagar - 608002

\begin{abstract}
India is considered to be the diabetic capital of the world. Almost $15 \%$ of the diabetics are prone for ulcers in their lifetime Diabetic foot ulcer remains a serious medical problem, which is extremely difficult to heal and exhibits a high recurrence rate. The major concern in this diabetic foot at present is the increasing incidence of multidrug resistant organisms. This study was done to know the prevalence, risk factors of MDRO and its impact on wound healing. The study sample was 150 diabetic foot patients admitted in surgery department, Rajah Muthiah Medical College, Chidambaram. In our study $66 \%$ of the ulcers grew multi-drug resistant organisms (MDRO) and 54.8\% of isolated organisms were multi drug resistant. Poor glycaemic control, previous hospitalisation, previous history of amputation, previous antibiotic usage, larger ulcer, necrotic ulcer, recurrent ulcers, higher grade of ulcer, presence of osteomyelitis, presence of retinopathy, peripheral vascular disease, neuropathy and polymicrobial culture, were significantly associated with MDRO infected foot ulcers. However, analysis by logistic regression revealed that only the recurrent ulcers and higher grade of ulcers were significantly associated with multi-drug resistant organism infections. The presence of MDRO does not have any impact on wound healing but it is associated with longer duration of hospital stay and increased rate of amputations.
\end{abstract}

Keywords: Diabetic foot, Multidrug resistant organism (MDRO).

\section{Introduction}

Diabetes mellitus is a chronic disease with chronic microvascular and macrovascular complications. India is considered by many, as the diabetic capital of the world. Like in other developing countries, complications of diabetic foot such as ulceration and infections, apart from causing high morbidity and mortality, also have social, and economic ramifications (Ako et al., 2006 ${ }^{1}$; Shankar et al., 2005² Gadepalle et al., $2006^{3}$ ). It has been reported that as high as $15 \%$ of all diabetics are prone to develop ulcers in their 
feet during their life time. Upto quarter of these patients will develop infections that will necessitate amputations. The major concern at present is the increasing incidence of multi-drug resistant organisms. The problem of multi-drug resistant organisms were poorly studied because of lack of uniform definitions and specific criteria to name an organism as multi-drug resistant. The European center for disease control and prevention has defined criteria, which are applicable universally. Very few studies have been done in India to analyse the prevalence and risk factors of multi-drug resistant organisms in relation to diabetic foot ulcers. The impact caused by multi-drug resistant organism were least analysed in Indian literature. Hence this study was done to analyse the prevalence, risk factors of multi-drug resistant organisms and its impact on wound healing in diabetic foot ulcers at a tertiary care hospital.

\section{Aim}

The primary objective was to study the prevalence, risk factors and impact of multi-drug resistant organism (MDRO) infection in diabetic foot ulcers.

\section{Methodology}

150 diabetic patients with foot ulcer were prospectively studied from Sep 2015 to Sep 2017 at Rajah Muthiah medical college, Annamalai University. Detailed clinical history and clinical examination of the ulcer were done for all patients. Patients were screened for neuropathy, nephropathy, retinopathy, peripheral arterial disease and underlying osteomyelitis using appropriate methods. The microbiological profile was analyzed in detail for each patient. MDRO are defined as microorganisms that are resistant to one or more classes of antimicrobial agents.

Infected ulcers were grouped into those with MDRO and those without MDRO and were then compared using univariate analysis. In order to identify the risk factor, for the presence of MDRO, analysis by logistic regression was done. Each patient was followed for a period of ten weeks to assess the status of wound healing. The impact of MDRO was assessed by analyzing the associations of amputations, duration of hospital stay, status of wound at ten weeks with MDRO infected ulcers using appropriate statistical tools. The influence of other factors on wound healing were analyzed by the same statistical tools.

\section{Results}

MDRO were isolated from 99 patients of 150 (66 $\%) .54 .8 \%$ (153 out of 279) of isolated organisms were multidrug resistant organisms. The commonest organism isolated in our study was Escherichia coli followed by Staphylococcus aureus and Pseudomonas aeruginosa. By univariate analysis poor glycaemic control, previous hospitalisation, previous history of amputation, previous antibiotic usage, size of ulcer, necrotic ulcer, recurrent ulcers, higher grade of ulcer, presence of osteomyelitis, presence of retinopathy, peripheral vascular disease, neuropathy and polymicrobial culture, were associated with significance in those with MDRO infected foot ulcers.

Analysis by Logistic regression indicated that, only two factors significantly increased the risk of acquiring MDRO infection; 1) Recurrent ulcer $(\mathrm{OR}=3.39, \mathrm{p}<0.05,95 \% \mathrm{CI}=[1.081-10.664])$, 2) Higher grade of ulcer ( $O R=13.44, \quad p<$ $0.001,95 \% \mathrm{CI}=[3.595-50.278]) . \quad$ It was found that the mean duration of hospital stay of patients with MDRO infections was 15.36 day ( $p$ $<0.001)$. MDRO in the foot ulcers significantly increased the frequency of amputations $(\mathrm{p}<0.01)$. MDRO infected ulcers had no impact on wound healing although they were significant by univariate analysis. By Logistic regression, age $(\mathrm{OR}=0.942, \mathrm{p}<0.1,95 \% \mathrm{CI}=[0.882-1.005])$, presence of PVD $(\mathrm{OR}=7.872, \mathrm{p}<0.01,95 \% \mathrm{CI}$ $=[2.009-30.849])$, osteomyelitis $(\mathrm{OR}=8.280$, $\mathrm{p}<0.01,95 \% \quad \mathrm{CI}=[1.768-38.766$ ]), nephropathy ( $\mathrm{OR}=4.36, \quad \mathrm{p}<0.05,95 \% \mathrm{CI}=$ [1.226 - 15.564$]$ ), inter-digital / digital ulcer ( OR $=0.073, \mathrm{p}<0.05,95 \% \mathrm{CI}=[0.006-0.869])$, elevated HbA1c ( OR $=6.020, \mathrm{p}<0.05,95 \% \mathrm{CI}$ $=[1.240-29.226])$, and higher Grade of ulcer 
$(\mathrm{OR}=4.10, \mathrm{p}<0.1,95 \% \mathrm{CI}=[0.863-19.549])$ significantly delayed wound healing.

\section{Discussion}

This study presents a comprehensive clinical and microbiological profile of infected diabetic foot ulcers, especially in relation to multidrug resistant organisms. With some reports of nearly $20 \%$ of hospital admissions being infected diabetic foot ulcers ${ }^{2}$ and with the growing global problem of multidrug resistant organisms ${ }^{4}$, we made an effort to study the role of multidrug resistant organisms in relation to diabetic foot ulcers.

In our study the foot ulcers were more prevalent in the fifth and sixth decade of life. The average age of the patients with foot ulcer was $58.21 \pm 9.3$ years. The foot ulcers were more common in male than female, which may be due to higher level of manual work and outdoor activity among male when compared to females.

Diabetic foot infection are usually polymicrobial in nature which is well documented in literature. In our study, $58.66 \%$ of ulcers had polymicrobial culture. In our study, the rate of isolation of organism per ulcer was 1.86 while the other two indianstudies (Mohammed et al ${ }^{5}$, Gadepalli ${ }^{3}$ ) showed a rate of 1.25 and 2.3 organisms per ulcer.

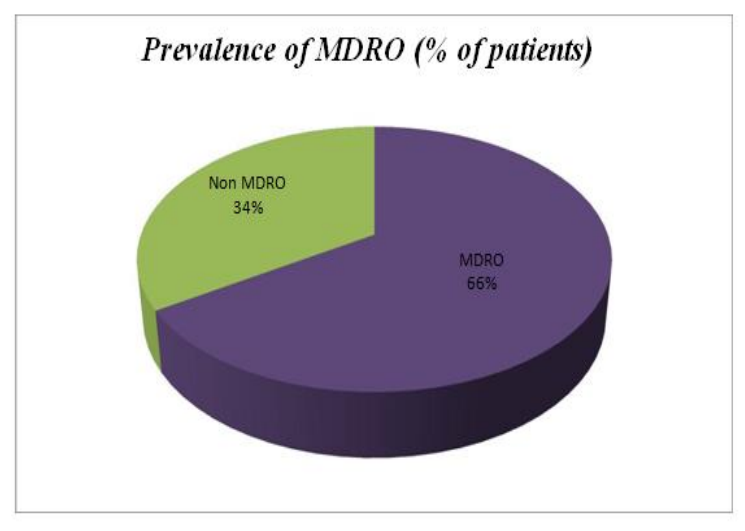

MDRO vs. Non MDRO (\% of isolates)



The commonest organism isolated in our study was Escherichia coli followed by Staphylococcus aureus, Pseudomonas and Klebsiella pneumoniae. In our study $66 \%$ of the ulcers grew multi-drug resistant organisms (MDRO) and $54.8 \%$ of isolated organisms were multi drug resistant. Apart from the multi drug resistant organisms like MRSA, ESBL, VRE which were extensively studied in literature, other groups of organisms like MDR Pseudomonas, Acinetobacter, Enterococcus, Enterobactereciae etc were also identified in our study. The higher degree of antibiotic resistance in tertiary care hospitals, could be because, with widespread usage of broad spectrum antibiotics, there occurs selective survival of drug resistant organisms. The increasing occurrence of MDROs is disconcerting because, infection with these organisms limits the choice of antibiotic treatment and may lead to a worse outcome.

Our study showed that $75 \%$ of all MDROs isolated were gram negative organisms. It is a fact that higher degree of antibiotic resistance is observed in gram negative organisms when compared to gram positive organisms. This is because gram negative organisms have a unique outer membrane which does not allow certain antibiotics to penetrate.

MRSA was seen in $18 \%$ of the patients in our study. $20.4 \%$ of isolated staphylococcus aureus were Methicillin resistant and coagulase negative (MRCONS), the reports of which in relation to diabetic ulcers were not looked at in the previous studies. With regard to the gram negative organisms in our study, E.coli showed greater antibiotic resistance, followed by Pseudomonas aeruginosa. $78 \%$ of isolated E.coli and $74 \%$ of isolated Pseudomonas were multi-drug resistant.

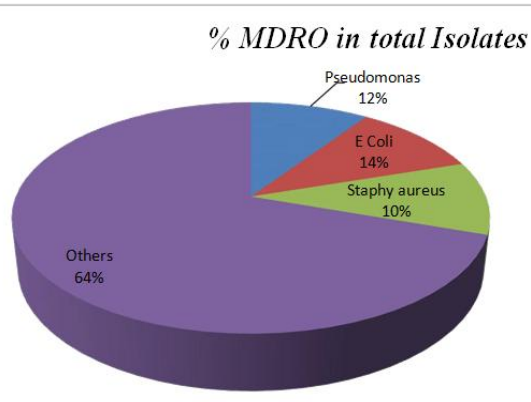


Among the isolated multi-drug resistant organisms, $25.49 \%$ were MDR E.coli, followed by $22.22 \%$ MDR Pseudomonas aeruginosa, 17.64 $\%$ methicillin resistant Staphylococcus aureus. Thus MDROs appear to be firmly entrenched in our patients, and posing questions to clinicians and microbiologists alike, with regard to patient management and the development of antibiotic policies.

In our study, univariate analysis showed that poor glycaemic control, previous hospitalisation, previous history of amputation, previous antibiotic usage, size of ulcer, necrotic ulcer, recurrent ulcers, higher grade of ulcer, presence of osteomyelitis, presence of retinopathy, peripheral vascular disease, neuropathy and polymicrobial culture, were significantly associated with MDRO infected foot ulcers.

However, analysis by logistic regression revealed that only recurrent ulcers and higher grade of ulcers were significantly associated with multi- drug resistant organism infections. It is possible that patients with recurrent ulcers had several courses of antibiotics, both during previous hospital admissions and from practitioners in the community, which led to resistance to multiple antibiotics. Higher grade of ulcers have an associated systemic sepsis and excessive local necrotic tissues.

Factors like previous hospitalization, previous antibiotic usage, poor glycemic control, ischemic ulcers have emerged as possible risk factors for MDRO in several other studies ${ }^{3,6}$. However, we have not found any significant association in our study.

In our study, the presence of MDRO in foot ulcers, significantly increased the duration of hospital stay and the associated cost. The mean duration of hospital stay in MDRO infected ulcer group was 15.36 days and that of non-MDRO group was 8.8 days.

Table 1: Mean duration of hospital stay

\begin{tabular}{|l|c|c|c|c|c|c|}
\hline \multirow{2}{*}{ MDRO } & \multirow{2}{*}{$\mathrm{N}$} & \multirow{2}{*}{ Mean } & \multicolumn{2}{|c|}{ Std. DeviationStd. Error } & \multirow{2}{*}{$\mathrm{t}$} & $\mathrm{P}$ \\
\cline { 4 - 5 } & & & \multicolumn{2}{|c|}{ Mean } & & value \\
\hline Non MDRO & 51 & 8.88 & 6.716 & .940 & & \\
\hline MDRO & 99 & 15.36 & 10.536 & 1.059 & 3.992 & 0.000 \\
\hline
\end{tabular}

Patients with MDRO had an increased rate of amputations both major and minor, in our study. Similar observations were found in the north Indian study (Mohammed Zubair et al ${ }^{5}$ ) and one from France (Richard et al ${ }^{6}$ ). We have seen that MDRO infections are associated with higher grade ulcers, and this could offer an explanation for the increased amputations.

Table 2: MDROs \& Amputations

\begin{tabular}{|l|c|c|c|c|c|}
\hline & $\begin{array}{c}\text { No } \\
\text { Amputation }\end{array}$ & Amputation & Total & $\mathbf{X}^{2}$ & P Value \\
\cline { 1 - 4 } NON & $40(78.4 \%)$ & $11(21.6 \%)$ & $51(100 \%)$ & \multirow{2}{*}{7.585} & 0.006 \\
\cline { 1 - 2 } MDRO & $55(55.6 \%)$ & $44(44.4 \%)$ & $99(100 \%)$ & & \\
\hline
\end{tabular}

We also made an effort to analyse the factors involved in determining the healing time of infected foot ulcers. We have found by multivariate analysis, the factors which determine the wound healing were the age, presence of peripheral arterial disease, osteomyelitis, nephropathy, interdigital ulcers, poorglycaemic control and grade of ulcer.

Although found significant by univariate analysis, the presence of MDRO had no role in determining the wound healing. This could be because of prompt change of antibiotics as dictated by the 
culture and sensitivity reports. Similarly other factors like smoking, size and depth of ulcers, duration of diabetes had no role in influencing the duration of wound healing. Similar observations were found in the study from France ${ }^{6}$, which also showed no role of MDRO in wound healing. The same study reported that the presence of neuroischaemic ulcers, proliferative retinopathy, and glycaemic control were the determining factors. Wound depth, presence of neuropathy and peripheral arterial disease have been reported to influence wound healing ${ }^{7,8}$.

\section{Conclusion}

The prevalence of MDRO is alarmingly high in infected diabetic foot ulcers. Recurrent ulcers and higher grade of ulcers are more prone to acquire MDROs. MDROs have no significant impact on wound healing. MDROs in diabetic foot ulcers are associated with longer duration of hospital stay and higher rates of amputations. Presence of peripheral arterial disease, osteomyelitis, nephropathy, inter-digital / digital ulcers, higher grade of ulcer and poor glycaemic control delays the healing of foot ulcer.

\section{References}

1. Ako-Nai AK, Ikem IC, Akinloye OO, Aboderin AO, Ikem RT, Kassim OO, . Characterization of bacterial isolates from diabetic foot infections in IleIfe, Southwestern Nigeria. The Foot, 2006; 16 (3): 158-164.

2. Shankar EM, Mohan V, PremalathaG, Srinivasan RS, Usha AR. Bacterial etiology of diabetic foot infections in South India. European Journal of Internal Medicine, 2005; 16: 567-570.

3. Gadepalli R, Dhawan B, Sreenivas V, Kapil A, Ammini AC, Chaudhry RA. Clinicomicrobiological study of diabetic foot ulcers in an Indian tertiary care hospital. Diabetes Care.2006 ; 29:17271732.
4. Wattal C. "Antibiotic policy": why and for whom. JIMSA 2004;17: 170-173.

5. Mohammad Zubair, Abiba Malik, Jamal Ahmad. Clinico-bacteriology and risk factors for the diabetic foot iinfection with multi-drug resistant microorganisms in North India., Biology and Medicine , 2010, vol2 (4): 22-34.

6. Richard JL, Sotto A, Jourdan N, et al., Risk factors and healing impact of multidrug-resistant bacteria in diabetic foot ulcers.Diabetes Metab. 2008 Sep; 34 (4 Pt 1):363-9.

7. Margolis DA, Allen - Taylor L, Hoffstad $\mathrm{O}$ et al ., Diabetic neuropathic foot ulccers? The association of wound size, wound duration and wound grade on healing. Diabetes care 2002; 25:1835-9.

8. Leese G, Schofield C, Mc Murray B et al., Scottish foot ulcer risk score predicts foot ulcer healing in a regional specialist foot clinic. Diabetes Care 2007; 30: 2064-9. 